\title{
Massive Ossification of the Patellar Fat Pad: An Unusual Cause of Knee Stiffness
}

\author{
Cara Cipriano, MD ${ }^{1}$ Nathan A. Mall, MD ${ }^{2}$ Michael J. Salata, MD ${ }^{3}$ Steven Gitelis, MD \\ Bernard R. Bach, Jr., MD ${ }^{1}$ \\ ${ }^{1}$ Department of Orthopaedic Surgery, Rush University Medical Center, \\ Chicago, Illinois \\ 2 Regeneration Orthopedics, St. Louis, Missouri \\ ${ }^{3}$ Department of Orthopedic Surgery, University Hospitals Case \\ Medical Center, Cleveland, Ohio \\ J Knee Surg Rep 2015;1:17-20. \\ Address for correspondence Bernard R. Bach, Jr., MD, 1611 W. \\ Harrison Street, Suite 300, Chicago, IL 60612 \\ (e-mail: brbachmd1952@gmail.com).
}

\begin{abstract}
Keywords

- heterotopic ossification

- knee mass

- arthrofibrosis

While stiffness following an injury is usually secondary to soft tissue scarring, a variety of different benign and malignant lesions may restrict range of motion about the knee. We report the unusual case of a patient who presented with a chronic knee extension contracture secondary to a large ossified mass in the anterior aspect of the knee. Radiographs, magnetic resonance imaging, and subsequent histopathology were consistent with a diagnosis of myositis ossificans of the fat pad. Subsequent resection resulted in full motion recovery and resolution of symptoms at 30-month follow-up.
\end{abstract}

Loss of knee motion following injury or surgery is frequently secondary to the development of soft tissue scarring intraand/or extra-articularly. As in cases of arthrofibrosis, loss of extension (flexion contracture) is typically related to scar tissue within the anterior intercondylar notch region, whereas loss of flexion (extension contracture) is usually secondary to scar within the suprapatellar pouch, medial gutter, lateral gutter, or a combination of these locations. ${ }^{1,2}$

Decreased motion is uncommonly associated with ectopic bone formation about the knee, which may take the form of heterotopic ossification (HO), myositis ossificans (MO), or neoplasm. ${ }^{2}$ This case report describes the evaluation and treatment of a patient who presented with massive MO formation in the fat pad region, producing an extension contracture, persistent pain, compensatory loss of patellar mobility, and impaired function. Excision of the ectopic bone mass resulted in resolution of symptoms and full motion recovery.

\section{Case Report}

A 46-year-old female patient presented complaining of pain and stiffness secondary to a mass in her left knee. She had sustained an isolated low energy crush injury
18 years ago, in which the knee was trapped between the bumper of a parked car and another automobile moving at approximately 1 to $2 \mathrm{mph}$. No fractures or specific injuries were noted, and she was allowed to continue her activities without restriction. The patient denied any history of head trauma. Her symptoms began 7 years following the injury with anterior knee swelling. She then noticed a mass on the medial aspect of her knee that progressed to involve the lateral side and was accompanied with increasing stiffness and progressive motion loss. She had been evaluated during this period and received reassurance that no operative treatment was required. However, she had not noticed any enlargement or sought further treatment in the 3 years before presentation (18 years postinjury).

Physical examination revealed a large, nontender bony mass located distal to and separate from the patella. This prominent mass extended medially, laterally, and anteriorly, with no masses noted posteriorly. It was not fixed to the skin but had minimal mobility. Range of motion was from 0 to 100 degrees of flexion with pain at terminal flexion. Patellar mobility was limited, and she had no extensor lag. The knee ligament examination was normal, she had no meniscal rotation signs, and there was no effusion. The extremity was neurovascularly intact with normal sensation about the knee. received

December 20, 2013 accepted after revision July 12, 2014 published online February 2, 2015
DOI http://dx.doi.org/ 10.1055/s-0034-1399760. ISSN 2326-2729.
Copyright $\odot 2015$ by Thieme Medical Publishers, Inc., 333 Seventh Avenue, New York, NY 10001, USA. Tel: +1(212) 584-4662.
License terms

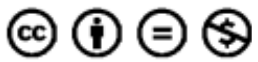



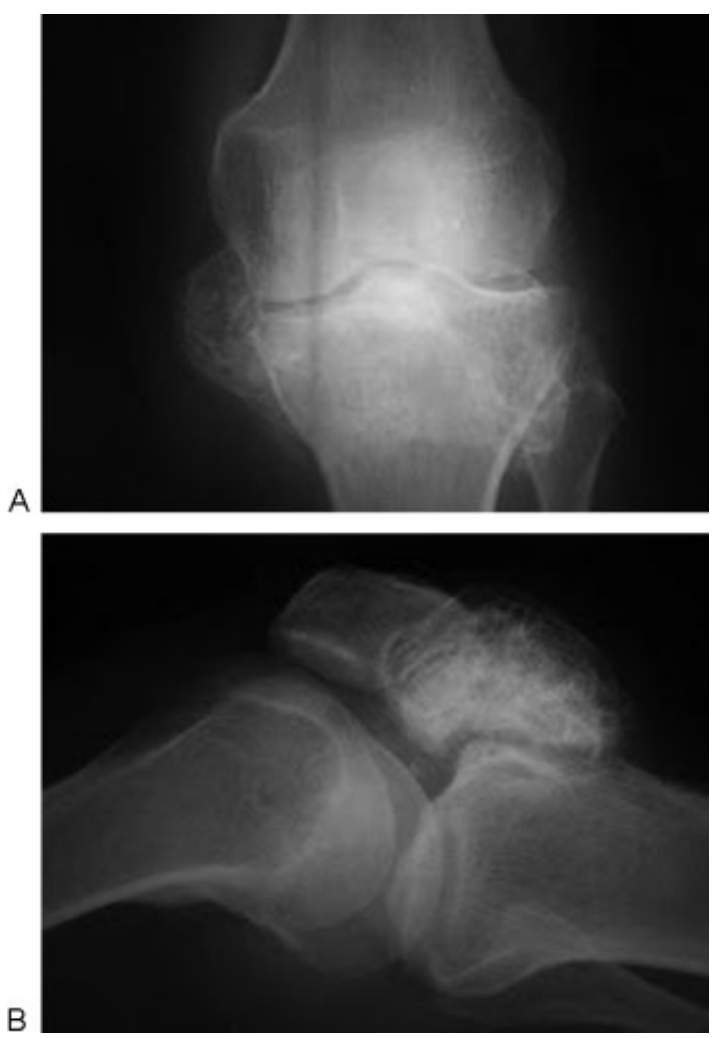

Fig. 1 (A, B) An anteroposterior and lateral radiograph of the knee reveals a large mature osseous mass extending medially, lateral, and posterior to the patellar tendon. There is no apparent involvement of the cortex.

Radiographs demonstrated an ossified mass inferior to the patella in the region of the fat pad (-Fig. 1A, B). Of note, findings suggestive of aggressive lesions such as periosteal reaction, radial ossification (often described as a "sunburst appearance"), or periosteal elevation ("Codman triangle") were absent. ${ }^{3}$ A magnetic resonance imaging was obtained to more accurately define the unusual location of the mass, which was found to be within the fat pad, clearly separate from the cortex as well as the patellar tendon (-Fig. 2). This characteristic helped to distinguish this from $\mathrm{HO}$, another form of benign bone formation that often occurs in injured or

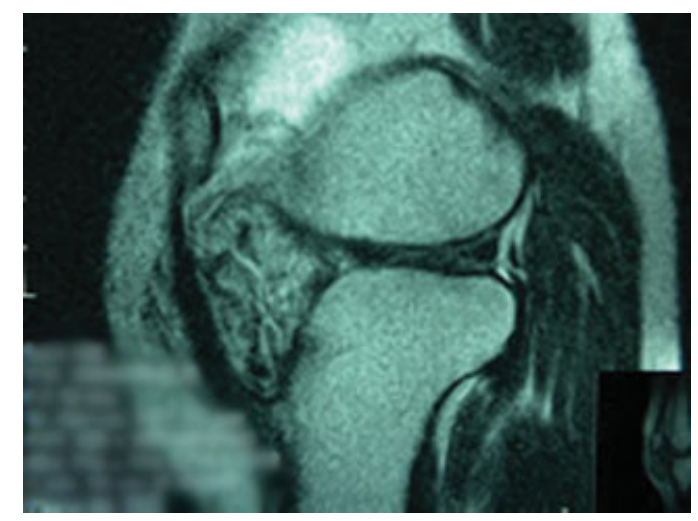

Fig. 2 Sagittal oblique magnetic resonance image of the knee demonstrating an osseous lesion that is separate from both the underlying cortex and patellar tendon.

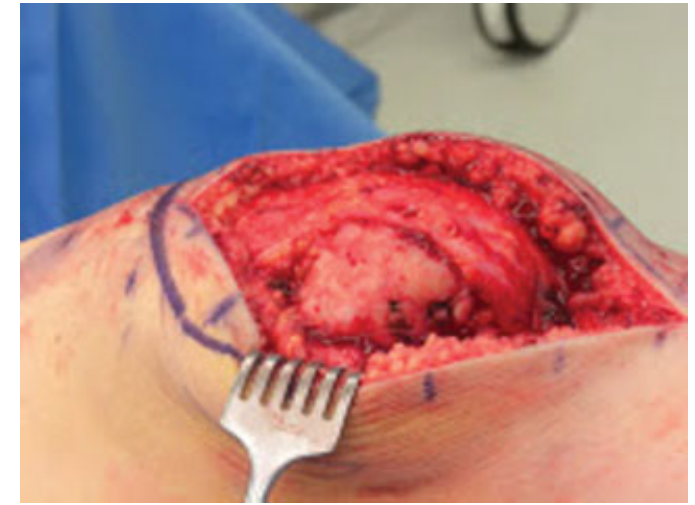

Fig. 3 An anteromedial arthrotomy performed through a longitudinal incision shows the underlying osseous mass in continuity posterior to the retinaculum.

spastic extremities; however, in contrast to MO, it is typically located between rather than within the planes of muscles or other soft tissue structures. ${ }^{4}$ Additionally, the patient was seen by our staff orthopedic oncologist who confirmed our clinical diagnosis. A biopsy was deferred because the characteristic appearance on radiographs and magnetic resonance imaging was consistent with heterotopic mature bone formation.

The mass was causing significant discomfort, deformity, and limited range of motion; as conservative management including knee range of motion exercises had failed to produce any improvement, the patient elected to undergo resection. Surgical exposure was achieved through an $8.5 \mathrm{~cm}$ midline incision carried down to the joint capsule where an anteromedial arthrotomy was performed. Upon opening the capsule the mass was immediately encountered below the retinaculum (-Fig. 3). Marsupialization was performed to expose the medial half of the $\mathrm{HO}$, and an elevator was used to separate it from the surrounding tissues. Segmental resection of the mass was then performed using a 0.75 -in. osteotome. The mass was removed posterior and lateral to the patellar tendon through this same exposure (-Fig. 4). Following excision of the mass, the knee could easily be flexed to

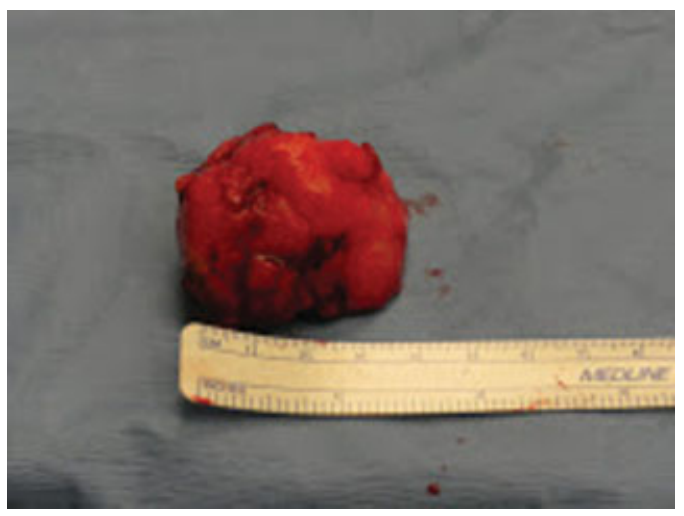

Fig. 4 The resected specimen is demonstrated. 
135 degrees. The patellar tendon was otherwise uninvolved and appeared to have maintained appropriate tension. The specimen was sent to pathology for histologic evaluation; both frozen and permanent section revealed mature lamellar bone without the malignant rimming osteoblasts that characterize osteosarcoma, thereby confirming the preoperative diagnosis of $\mathrm{MO}$ (-Fig. 5$)^{4}$

The patient tolerated the procedure well and was allowed to progress as tolerated in terms of weight-bearing, range of motion, and straight leg raises. Indomethacin $75 \mathrm{mg}$ (Indocin, Merck \& Company Inc., West Point, PA) was prescribed for 6 weeks postoperatively to prevent recurrence, and physical therapy was started immediately and was directed at motion recovery and closed chain quadriceps strengthening. At 6 weeks postoperatively, she had active range of motion from full extension to 135 degrees of flexion without an extensor lag (-Fig. 6).

At her most recent clinic visit (30 months postoperatively), she has remained active without restriction. Her range of motion was from 3 degrees of hyperextension to 140 degrees of flexion and is symmetric with the contralateral extremity. She was able to perform a straight leg raise without a lag, and had approximately $1 / 4$ in smaller thigh girth than the uninvolved extremity. She had no knee pain, swelling or functional limitations. Radiographs revealed no evidence of recurrent ossification. The patient was extremely satisfied.

\section{Discussion}

Several forms of ectopic ossification may occur around the knee. Small ossicles may form in the patellar tendon as a result of Osgood-Schlatter disease, Jumper knee, or

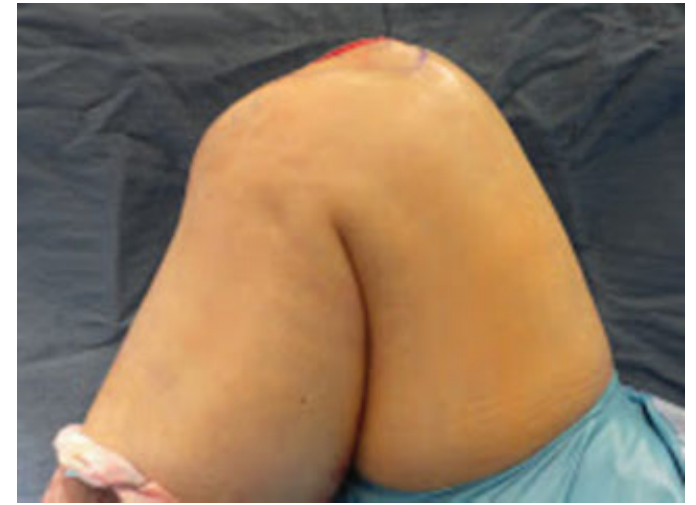

Fig. 6 Photograph taken at 6 weeks after the surgery, demonstrating the patient with 135 degrees of knee flexion.

Sinding-Larson-Johansson syndrome. ${ }^{5-7}$ More extensive ossification of the patellar tendon can develop in the posttraumatic setting. Matsumoto et al described two cases of tendon ossification in patients with patella alta, which they speculate resulted from prior tibial tuberosity or patellar sleeve fracture. ${ }^{8}$ We have also noted ossification within the patellar tendon after grafting the distal patellar defect following the use of a bone patellar bone autograft for anterior cruciate ligament surgery. ${ }^{9}$

$\mathrm{HO}$ is another benign form of ectopic ossification that, such as $\mathrm{MO}$, frequently develops following local trauma. It is also associated with muscle spasticity secondary to neurologic damage, including brain injury, spinal cord injury, and other upper motor neuron lesions. ${ }^{7} \mathrm{HO}$ is typically found between the muscles surrounding joints rather than within the
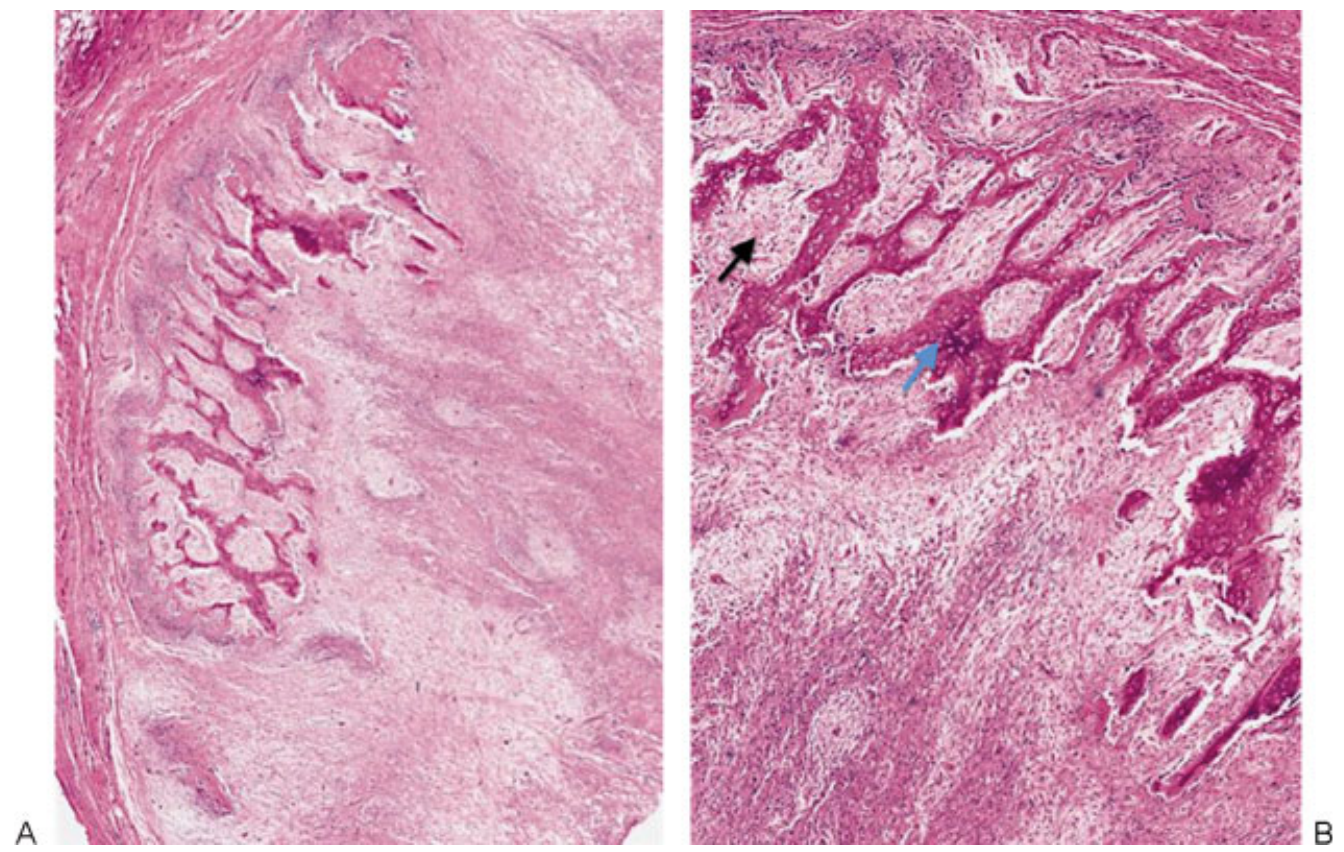

Fig. 5 Low power (A) and high power (B) histologic specimens of myositis ossificans. These photographs demonstrate the typical zonation consisting of peripheral osteoid and scattered mature woven bone (blue arrow) surrounding central myxoid matrix (black arrow) and uniform fibroblasts with faintly eosinophilic cytoplasm. 
muscles or joints themselves. Around the knee, HO is most commonly seen beneath the quadriceps or associated with the medial collateral ligament. ${ }^{7}$ Radiographically it appears as bone spurs or islands that may eventually grow to forming bridges or large masses, and it is histologically similar to MO. Surgical excision is indicated for symptomatic lesions only after they reach maturity, which usually occurs in the first 6 months when it is posttraumatic but may take over a year when neurologic in origin.

Far less common, and especially unlikely in the absence of pain or rapid progression, osteosarcoma may occur extraosseously, most often in the areas of the buttock or proximal thigh. ${ }^{4}$ Plain radiographs remain the imaging study of choice and may reveal signs of aggressive behavior as described above within the case report. Of note, the ossification of osteosarcoma begins centrally, while the benign ossification of MO is initially seen at the periphery of the lesion, which consolidates inward as it matures. ${ }^{10}$ If symptoms or imaging are suspicious for malignancy, consultation with an orthopedic oncologist would be the next most appropriate step in diagnosis and management. A biopsy might then be considered, followed by the appropriate treatment; in the case of sarcoma, this would consist of neoadjuvant chemotherapy, wide excision, and further chemotherapy. ${ }^{4}$

The ectopic bone in our case is best classified as MO, which describes bone formation within soft tissue structures such as striated muscle, ligaments, tendons, and fascia. Most commonly, this occurs as a solitary lesion and is posttraumatic (posttraumatic myositis ossificans), which accounts for 60 to $75 \%$ of the cases. ${ }^{9}$ Patients are typically in their second or third decade. ${ }^{9,11}$ It most frequently affects skeletal muscle on the proximal, anterior aspect of the limbs, especially the vastus lateralis in the thigh and the brachialis in the arm. ${ }^{12}$ The lesions are often painful, tender, and erythematous, especially in the early stages, with joint contractures typically complicating chronic cases. Early radiographs may be negative or demonstrate a subtle osseous reaction; at 1 week, peripheral calcifications begin to form, and by 2 months a well-defined cortical rim can be seen peripherally, and more mature lesions may develop internal calcifications as well. The radiographic hallmark of MO is that the lesion is separated from the cortex; histology it appears identical to normal bone but lacks a periosteal layer. ${ }^{4}$ Treatment is symptomatic, with operative intervention only directed at relieving pain or other complications of mass effect. Resection should be performed only after the lesion reaches maturity, which may be recognized by the presence of sharp cortical margins on radiographs. Although this time period was traditionally believed to be 12 to 18 months postinjury, recent literature suggests it may be as small as 3 to 5 months. ${ }^{13}$

Although the exact pathophysiology remains unclear, evidence suggests that a relationship exists between different form of benign ectopic bone formation, as well as a genetic predisposition for these disorders in certain individuals. Indomethacin (6 weeks) or radiation therapy (single dose administered within 72 hours of trauma or surgery) are commonly used as prophylaxis in individuals at high risk for ectopic bone formation or recurrence. Most of the data supporting this treatment is derived from studies on $\mathrm{HO}$ in patients following total hip arthroplasty or traumatic brain and spinal cord injury. ${ }^{12}$ In our case, the patient's initial bone formation was the result of a dramatic response to relatively minor trauma, indicating a potential susceptibility to further bone formation. Because of this, as well the low probability of adverse effects from prophylaxis, Indomethacin was prescribed postoperatively to minimize the risk of recurrence.

Significant MO of the fat pad has, to our knowledge, not previously been described. The case is unusual in that the patellar tendon was uninvolved, making it possible to excise the mass without reconstructing the tendon. This resulted in an excellent clinical result, with immediately improved range of motion and preservation of extensor mechanism function postoperatively. This motion was maintained at final followup, her function improved significantly, and her pain at the end range of motion was eliminated.

\section{References}

1 Petsche TS, Hutchinson MR. Loss of extension after reconstruction of the anterior cruciate ligament. J Am Acad Orthop Surg 1999; 7(2):119-127

2 Chen MR, Dragoo JL. Arthroscopic releases for arthrofibrosis of the knee. J Am Acad Orthop Surg 2011;19(11):709-716

3 Enneking WF, Rathe R, Cornwall G. Clinical Musculoskeletal Pathology. Gainesville, FL: Office of Medical Informatics, College of Medicine, University of Florida; 1998

4 Cipriano CA, Pill SG, Keenan MA. Heterotopic ossification following traumatic brain injury and spinal cord injury. J Am Acad Orthop Surg 2009;17(11):689-697

5 Bruijn JD, Sanders RJ, Jansen BR. Ossification in the patellar tendon and patella alta following sports injuries in children. Complications of sleeve fractures after conservative treatment. Arch Orthop Trauma Surg 1993;112(3):157-158

6 Bowers KD Jr. Patellar tendon avulsion as a complication of Osgood-Schlatter's disease. Am J Sports Med 1981;9(6):356-359

7 Grossfeld SL, Engebretsen L. Patellar tendinitis-a case report of elongation and ossification of the inferior pole of the patella. Scand J Med Sci Sports 1995;5(5):308-310

8 Matsumoto H, Kawakubo M, Otani T, Fujikawa K. Extensive posttraumatic ossification of the patellar tendon. A report of two cases. J Bone Joint Surg Br 1999;81(1):34-36

9 Tornetta P III, Barbera C. Severe heterotopic bone formation in the knee after tibial intramedullary nailing. J Orthop Trauma 1992; 6(1):113-115

10 Tyler P, Saifuddin A. The imaging of myositis ossificans. Semin Musculoskelet Radiol 2010;14(2):201-216

11 Hanquinet S, Ngo L, Anooshiravani M, Garcia J, Bugmann P. Magnetic resonance imaging helps in the early diagnosis of myositis ossificans in children. Pediatr Surg Int 1999;15(3-4): 287-289

12 Hait G, Boswick JA Jr, Stone NH. Heterotopic bone formation secondary to trauma (myositis ossificans traumatica). J Trauma 1970;10(5):405-411

13 Hosalkar H, Pandya NK, Hsu JE, Kamath AF, Keenan MA. What's new in orthopaedic rehabilitation. J Bone Joint Surg Am 2010; 92(8):1805-1812 